\title{
Point Pattern Analysis of Different Life Stages of Haloxylon ammodendron in Desert-oasis Ecotone
}

\author{
Mei Wang', Shuoxin Zhang', Guangming $\mathrm{Chu}^{1 *}$ \\ 'Department of Forestry, Agricultural College, Shihezi University, Shihezi, Xinjiang 832000, China \\ ${ }^{2}$ College of Forestry, Northwest A\&F University, Yangling 712100, China
}

Received: 2 April 2014

Accepted: 8 July 2014

\begin{abstract}
The Haloxylon ammodendron natural desert forest is an important vegetation formation and has a large distribution area in Junggar Basin, Xinjiang Province, China. In this paper, H. ammodendron populations were mapped, characterized and the spatial distribution patterns and spatial associations of $H$. ammodendron among different life stages (seedlings $-\mathrm{S}$, juveniles $-\mathrm{J}$, mature $-\mathrm{M}$, overmature $-\mathrm{O}$ ) were analyzed using $O$-ring univariate $O_{11}(r)$ and bivariate $O_{12}(r)$ statistics. We found that:

(1) H. ammodendron was a regenerating population.

(2) H. ammodendron (S), H. ammodendron (J), and H. ammodendron (M) showed significant aggregations at scale 0-8 m, 0-27 m, and 0-35 m, respectively, whereas H. ammodendron (O) tended to have random distributions at almost all scales.

(3) The spatial associations between different life stages of H. ammodendron tended to turn from positive to negative if their size differences are enlarged increasingly.

Strongly positive associations were found at small scales for only two pairs: $H$. ammodendron (S)-H. ammodendron (J), H. ammodendron (J)-H. ammodendron (M).
\end{abstract}

Keywords: Haloxylon ammodendron, spatial pattern, spatial association, Junggar Basin

\section{Introduction}

Stand structure is known to interact with growth, survival, density, and spatial patterns to influence competition and demographic changes in a population [1-3]. Furthermore, forest stand structure is a key element in understanding forest ecosystems [4]. At a certain extent, stand structure determines habitat and species diversity and can be quantified to assess habitat quality for conservation purposes $[5,6]$. In addition, one of the major components of forest stand structure is spatial pattern [7]. Therefore, the study of tree spatial patterns in forest stands also has

*e-mail: chgmxj@126.com become a relevant tool in the analysis of the structure and dynamics of forest communities [5].

Ecologists study spatial patterns of species at different life stages to infer the existence of underlying processes that have generated these patterns and identify the scale at which those processes are operating [7]. Analyzing spatial distributions of individuals within life stages and spatial associations between different life stages is essential for understanding the spatial and temporal dynamics of populations [8]. Thus, it is significant to analyze the population regeneration and intraspecific relationships among these life stages in forests.

Haloxylon ammodendron (C.A. Mey.) Bunge (Chenopodiaceae), a sub-tree xerophilous plant, dominates 
Table 1. Stand structure and composition in the plot.

\begin{tabular}{|l|c|c|c|c|c|c|}
\hline \multicolumn{1}{|c|}{ Species } & $\begin{array}{c}\text { Living standing } \\
{[\text { No./ha] }}\end{array}$ & $\begin{array}{c}\text { Dead adult standing } \\
{[\text { No./ha] }}\end{array}$ & $\begin{array}{c}\text { Average height } \\
{[\mathrm{cm}]}\end{array}$ & $\begin{array}{c}\text { Max. height } \\
{[\mathrm{cm}]}\end{array}$ & $\begin{array}{c}\text { Average canopy } \\
{[\mathrm{cm}]}\end{array}$ & $\begin{array}{c}\text { Max. canopy } \\
{[\mathrm{cm}]}\end{array}$ \\
\hline H. ammodendron & $1711 \pm 306 \mathrm{a}$ & $92 \pm 19 \mathrm{~b}$ & $93.1 \pm 86.7 \mathrm{a}$ & $430.5 \pm 15.4 \mathrm{a}$ & $104.7 \pm 47.9 \mathrm{a}$ & $250.3 \pm 31.9 \mathrm{a}$ \\
\hline A. aphylla & $1273 \pm 206 \mathrm{~b}$ & $715 \pm 219 \mathrm{a}$ & $43.9 \pm 21.4 \mathrm{c}$ & $110.2 \pm 8.6 \mathrm{~b}$ & $47.3 \pm 20.2 \mathrm{c}$ & $105.7 \pm 13.6 \mathrm{c}$ \\
\hline R. songarica & $183 \pm 18 \mathrm{c}$ & $27 \pm 18 \mathrm{c}$ & $37.6 \pm 17.5 \mathrm{~d}$ & $55.7 \pm 7.2 \mathrm{e}$ & $39.7 \pm 14.8 \mathrm{e}$ & $86.8 \pm 21.6 \mathrm{~d}$ \\
\hline N. roborowskii & $126 \pm 15 \mathrm{~d}$ & $18 \pm 11 \mathrm{~d}$ & $49.5 \pm 23.1 \mathrm{~b}$ & $80.4 \pm 5.3 \mathrm{~d}$ & $77.4 \pm 25.6 \mathrm{~b}$ & $220.1 \pm 46.3 \mathrm{a}$ \\
\hline Others & $53 \pm 17 \mathrm{e}$ & $12 \pm 6 \mathrm{e}$ & $44.2 \pm 7.8 \mathrm{c}$ & $96.2 \pm 20.7 \mathrm{c}$ & $42.5 \pm 12.4 \mathrm{~d}$ & $140.5 \pm 35.7 \mathrm{~b}$ \\
\hline
\end{tabular}

many areas of Asian deserts with average annual rainfall between 30 and $200 \mathrm{~mm}$. In China, H. ammodendron is an important component of old Mediterranean flora widely spread in the Junggar Basin, northeast of the Tarim Basin and several other desert areas on a range of soils $[9,10]$. This species is of great ecological and economic importance not only because it can survive harsh environmental conditions, but it can also stop wind erosion of sand. Song et al. developed some studies on the special pattern of H. ammodendron population, and suggested that aggregated distribution is the main trend for the $H$. ammodendron population for adaptation to the unfavorable environment, which is beneficial to the survival and development of the individual [11].

To address these concerns, we studied the spatial patterns of $H$. ammodendron at different life stages (height classes) with the $O$-ring statistic, and mainly focused on intraspecific spatial associations among the life stages in a 3 ha desert plant plot in the desert-oasis ecotone of south Junggar Basin, NW China. Our objectives were:

(1) To detect the dynamic aspects of spatial pattern at different life stages (seedlings, juveniles, mature, and overmature)

(2) To reveal the spatial association within different life stages among seedlings, juveniles, mature, and overmature

Thus our study provides a theoretical basis for the conservation, rehabilitation and sustainable management of desert ecosystem in Junggar Basin.

\section{Materials and Methods}

\section{Study Site}

The study area was located on the desert-oasis ecotone spanning an elevation range of 258-265 $\mathrm{m}$ in South Junggar Basin, NW China $\left(45^{\circ} 22^{\prime} 43.4^{\prime \prime} \mathrm{N}, 84^{\circ} 50^{\prime} 32.5^{\prime \prime} \mathrm{E}\right)$, from July to September 2010, which is a transitional zone from oasis to desert (Fig. 1). Geological substrates of the study site include aeolian deposits of sandy soil and highly eroded diluvial soil. The mean temperature varies from 5 to $9^{\circ} \mathrm{C}$, minimum winter temperatures vary from -30 to $-41^{\circ} \mathrm{C}$, and maximum summer temperatures are $30-40^{\circ} \mathrm{C}$. Snow melt at the end of winter, together with rainfall, amounts to an annual precipitation of $100-150 \mathrm{~mm}$.

Three typical sample plots (3 ha) were laid out in an area with ecotone or hierarchical distribution between dilu- vial and sandy soil. Sandy soil area is about $55-65 \%$ and sand layer is $5-30 \mathrm{~cm}$ deep. The micro topography of three plots is complicated and heterogeneous. In the study area, the vegetation cover ranges between $10 \%$ and $35 \%$. The woody vegetation is dominated by $A$. aphylla, H. ammodendron, Reaumuria songarica, and Nitraria roborowskii (Table 1). Other woody plant species in the area include Lycium ruthenicum, Halostachys caspica, Halocnemum strobilaceum, and Tamarix ramosissima.

\section{Data Collection}

Each plot $(100 \times 100 \mathrm{~m})$ was divided into 400 contiguous $5 \times 5 \mathrm{~m}$ quadrates, as the basic unit of vegetation survey, using the DQL-1 forest compass (Harbin Optical Instrument Factory, China). All woody plants, including living and dead standing ones, were investigated. The species names, relative location of each individual, height, crown width (the greatest diameter of the vertical projection of the crown in two directions, i.e., north-south or east-west), and microhabitat (e.g., soil surface, depression patch) were recorded.

Since spatial patterns at each life stage suggests the past process of regeneration [12], we classified $A$. aphylla plants based on reproductive ability into seedlings (height $\leq 50$ $\mathrm{cm})$, juveniles $(50<$ height $\leq 150 \mathrm{~cm})$, mature $(150<$ height $\leq 250 \mathrm{~cm}$ ), and overmature (height $\geq 250 \mathrm{~cm}$ ).

\section{Point Pattern Analysis}

Wiegand and Moloney developed a multiscale method called the $O$-ring statistic that is based on the $L$-function [13]. The $O$-ring statistic isolates specific distance classes

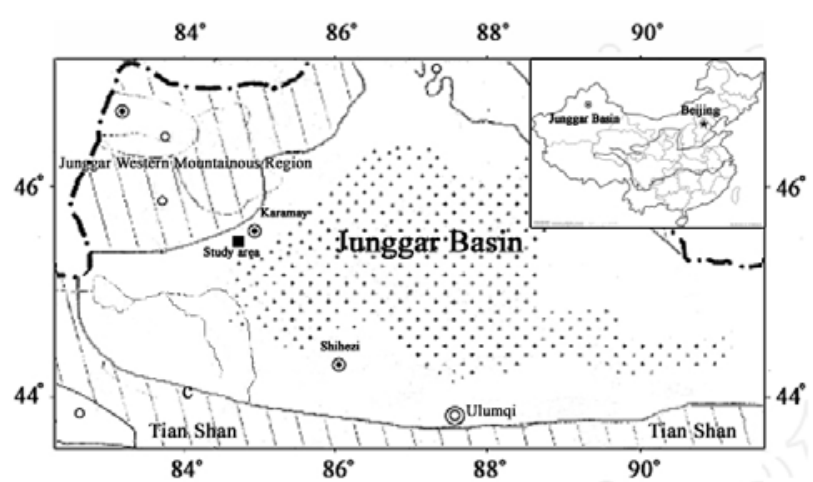

Fig. 1. Location map of study area. 
by replacing the circles of Ripley's $K$-function with rings, and by using the mean number of neighbors in a ring of radius $\mathrm{r}$ and ring width around an individual [13]. Consequently, the $O$-ring statistic has the additional advantage that it is a probability density function with the interpretation of a neighborhood density, which is more intuitive than an accumulative measure [14]. For the $O$-ring statistic, the univariate statistic is used to analyze the spatial pattern of one object, while the bivariate statistic is used to analyze the spatial association of two objects (pattern 1 and pattern 2) [13].

In this study, the univariate $O$-ring statistic was used to analyze the spatial patterns of different life stages of plants, and the bivariate $O$-ring statistic was used to analyze spatial associations among different life stages in the three plots. For the univariate analyses we used the null model of complete spatial randomness (CSR) as a null hypothesis [13, 15] because the spatial distributions of plants in the three plots seem to be affected significantly by drought stress and habitat heterogeneity (e.g., soil patch and micro topography). To test intraspecific association we examined the spatial distribution relation among four life stages of $H$. ammodendron plants with the antecedent condition null model. In this case, the locations of pattern 1 remain fixed and the null model distributions only pattern 2 in accordance to a specific univariate null model [15]. For univariate analysis, $O(r)$ above the upper confidence limit indicates aggregation while $O(r)$ below the lower confidence limit indicates regularity. For bivariate analysis, if at a given distance r, $O_{12}(r)$ is above the upper (or below the lower) limit of the confidence envelope, it indicates that life stage 2 is positively (or negatively) associated with life stage 1 at the distance r. $O_{12}(r)$ is within the confidence intervals, which indicates that there is no interaction between life stages 1 and 2 .

In addition, the intensity of spatial patterns or spatial associations is defined as the magnitude of the deviation from randomness or independence. All analyses were conducted in the Programita software package [13]. Ninetynine Monte Carlo simulations were used to generate $99 \%$ confidence envelopes.

\section{Result}

\section{Stand Structure and Composition}

The total number of woody plants in the plot was 4,210 individuals $\cdot$ ha $^{-1}$ (3,346 living and 864 dead standing), consisting of 8 species, 8 genus, and 3 families. H. ammodendron population was in possession of the dominance of spatial occupation capacity in the plot, since 1,803 individuals or $42.8 \%$ were $A$. aphylla (1,711 live and 92 dead standing) (Table 1). A. aphylla (1,273 live and 715 dead standing) was the main associated plant species of $H$. ammodendron. The other species together occupied only $10 \%$ of the total number. $H$. ammodendron was the most abundant canopy species and showed the largest mean canopy $(104.7 \mathrm{~cm})$ and largest mean height $(93.1 \mathrm{~cm})$.
The largest plant height measured in the plot was for $H$. ammodendron $(4.3 \mathrm{~m})$, and the other species was not more than $1.2 \mathrm{~m}$. H. ammodendron population in the plot exhibited reverse-J plant height distributions due to a large number of small size plants, characterized by continuous regenerating populations (Fig. 2).

\section{Spatial Patterns of Different Life Stages}

Maps of tree locations of the H. ammodendron across four life stages were plotted (Fig. 3). The maps clearly showed that $H$. ammodendron is not uniform across the plot. When the spatial patterns of $H$. ammodendron was analyzed at four life stages (Fig. 4). H. ammodendron (S), $H$. ammodendron $(\mathrm{J})$, and $H$. ammodendron $(\mathrm{M})$ showed significant aggregations at scale $0-8 \mathrm{~m}, 0-27 \mathrm{~m}$, and 0-35 m, respectively. $H$. ammodendron $(\mathrm{O})$ tended to have random distributions at all scales and only had a fluctuation around the upper envelope line at small scales. However, the aggregated intensity decreased from $H$. ammodendron (S) to $H$. ammodendron (M).

\section{Spatial Association Among Life Stages}

H. ammodendron (S) had spatially positive association with $H$. ammodendron $(\mathrm{J})$ at $0-4 \mathrm{~m}$ scales, and negative association at 9-37 m scales (Fig. 5a). H. ammodendron (S) was significantly and negatively associated with $H$. ammodendron (M) at 0-25 m scales, and tended to have independent associations with a fluctuation around the upper envelope line at $>25 \mathrm{~m}$ scales (Fig. 5b). H. ammodendron (S) and $H$. ammodendron $(\mathrm{O})$ tended to have spatially no association, except positive association at 3-6 m and 7-20 m scales (Fig. 5c). H. ammodendron (J) was significantly and positively associated with $H$. ammodendron (M) at 0-29 m scales, and tended to have independent associations with a fluctuation around the lower envelope line at $>29 \mathrm{~m}$ scales (Fig. 5d). H. ammodendron (J) and H. ammodendron (O) showed no association at $0-16 \mathrm{~m}$ scales, and mainly negative association at middle scales (Fig. 5e). H. ammodendron (M) was dominated by spatial independence with $H$. ammodendron (O) at almost all scales (Fig. 5f).

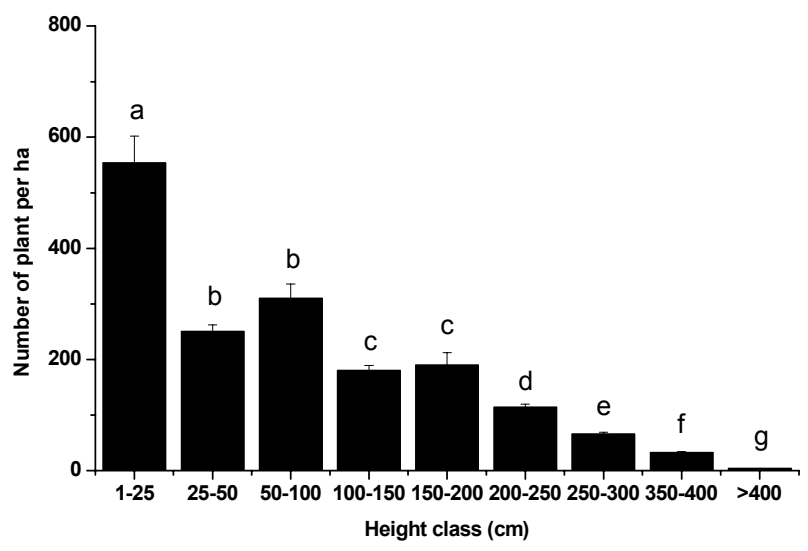

Fig. 2. The height classes distribution of H. ammodendron population in the plot. 
(a) Seedlings

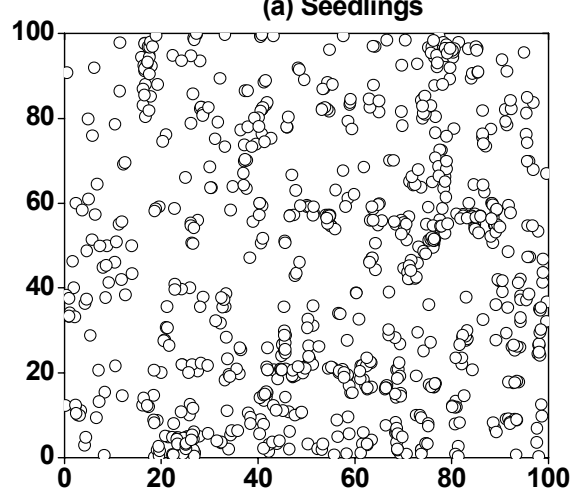

(c) Mature

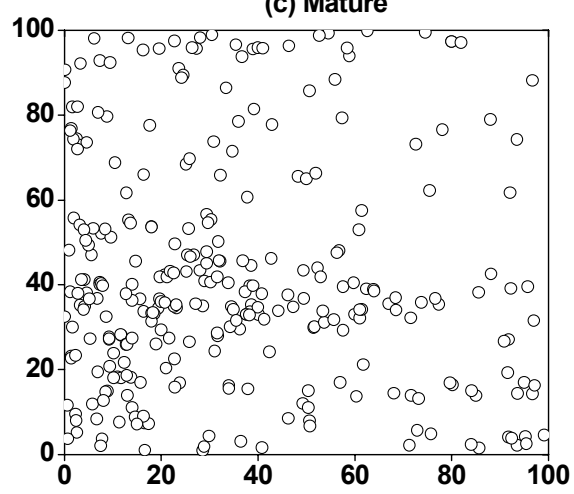

(b) Juveniles

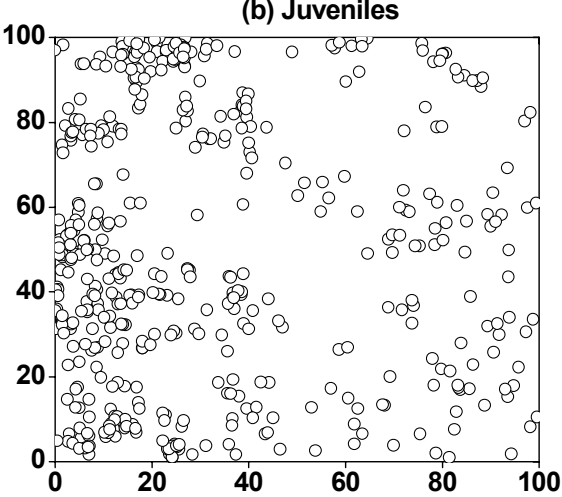

(d) Overmature

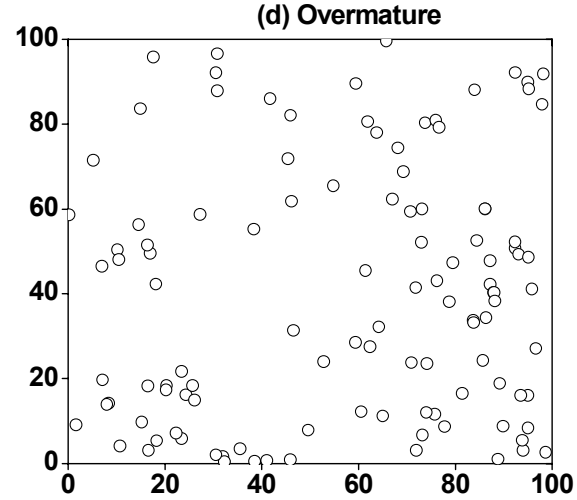

Fig. 3. The distribution maps of H. ammodendron plants at four life stages.

\section{Discussion}

Population Structure

The size structure of a species reflects regeneration processes $[16,17]$, and when compared to the spatial structure of forest, can provide insight into the forest dynamics [18]. H. ammodendron plants in the plot had a large number of seedlings, and the size distribution of H. ammoden- dron population appears as a reversed "J" shape, suggesting the continuous population regeneration and favorable conditions for establishment and survival of seedlings. The less number of $H$. ammodendron (O) might be offset by the younger $H$. ammodendron (M). Meanwhile, they also have a certain number of $H$. ammodendron $(\mathrm{J})$ and may be able to maintain the population in desert-oasis ectone.

However, $\mathrm{Si}$ et al. reported H. ammodendron population has degenerated in low-lying areas and small dunes
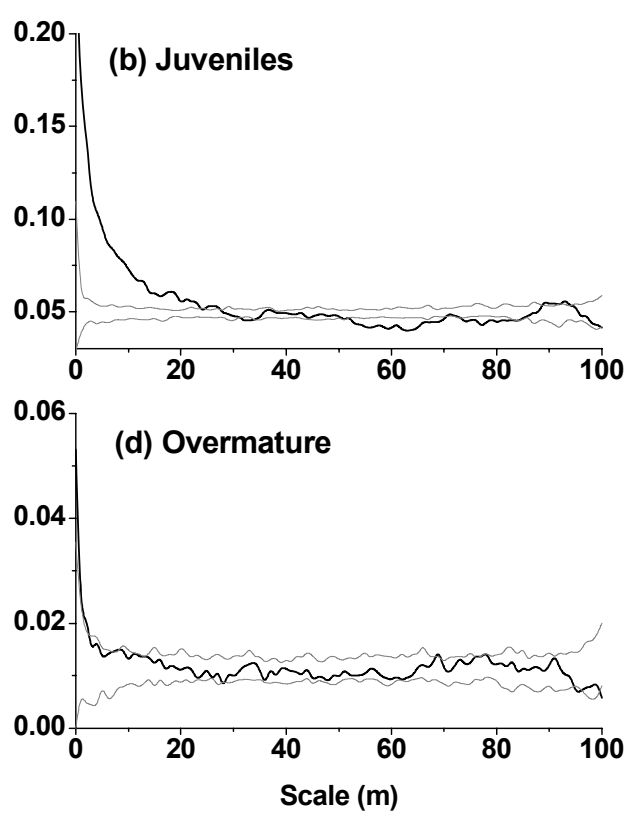

Fig. 4. Spatial patterns of $H$. ammodendron at four life stages with univariate $O$-ring. Black lines indicate ring statistics $(O(r))$; thindashed lines indicate the upper and lower limits of the $99 \%$ confidence envelope. 

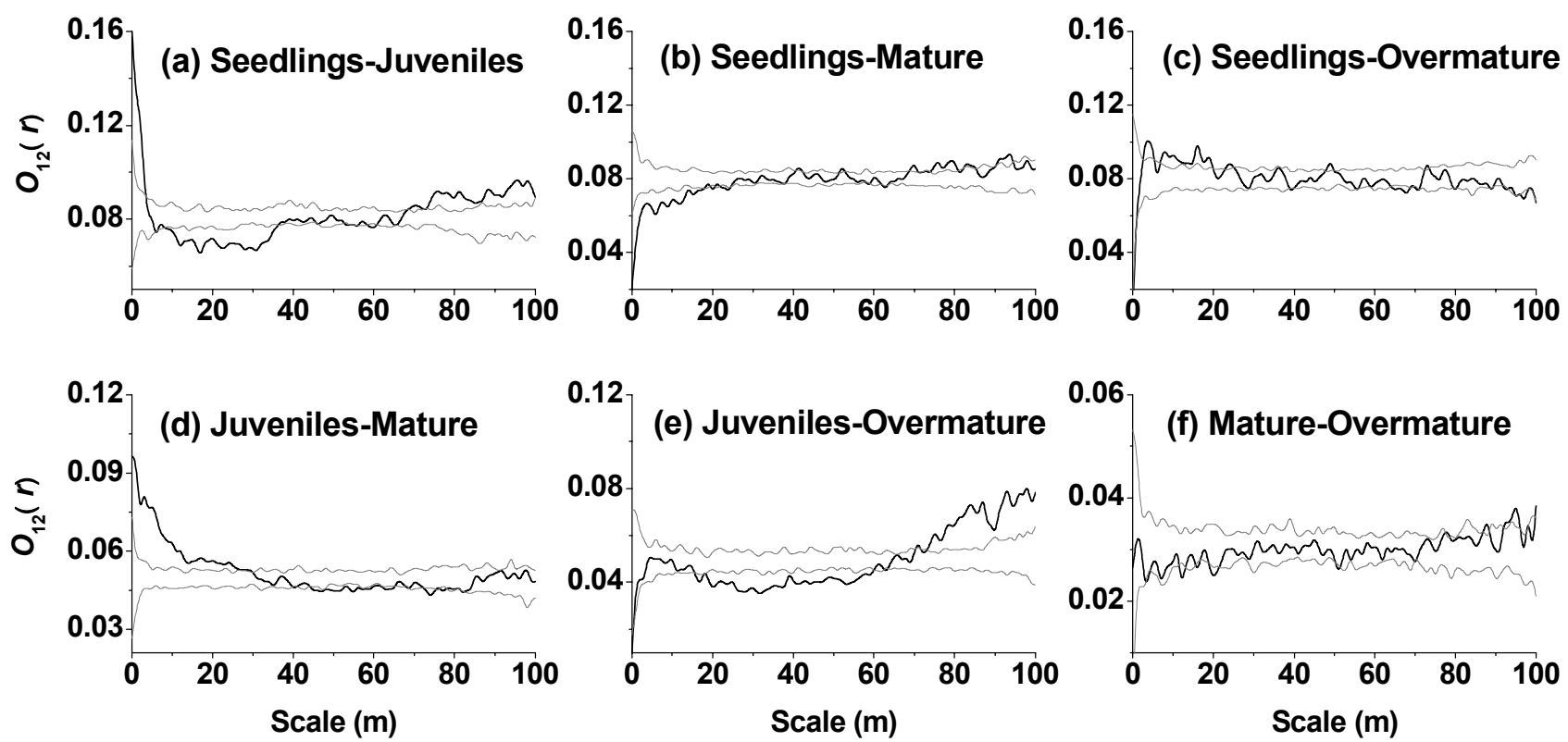

Fig. 5. Spatial associations of different life stages. Black lines indicate ring statistics $(O(r))$; thin-dashed lines indicate the upper and lower limits of the $99 \%$ confidence envelope.

(height $<5 \mathrm{~m}$ ), while nearby in large dunes (height $>10$ $\mathrm{m})$ the plants grow normally [19]. On the other hand, the groundwater depth has severely restricted the availability of water to $H$. ammodendron and seedling regeneration in the degraded area [10]. The relationship between the survival index of $H$. ammodendron population and the groundwater level was significantly negative, indicating that groundwater has a profound effect on the viability of the populations [19]. In the present study, but although large dune is nonexistant, $H$. ammodendron renewal is normal. Thus, we suggested that groundwater depth with that between 4-6 m is the effective degree for $H$. ammodendron growth and seedlings regeneration. In addition, $H$. ammodendron is a psammophytes species and only dungarunga in Gurbantunggut Desert, and have strong drought-tolerant and barren resistance traits [10]. The species can produce a large number of seeds, and the seedlings have rapid growth and lignifications phenomena after spring snowmelt in order to adapt specific desert habitats. Their biological characteristics and reproduction in local habitats provide sufficient opportunities for establishment and recruitment, and therefore $H$. ammodendron grows well relative to other species in the study area.

\section{Spatial Patterns}

Aggregated distribution in species is a widespread pattern in nature $[7,20]$. Likewise, this issue is especially relevant and common in semi and semi-arid shrub lands [11, 21, 22]. Aggregated plant patterns in semiarid shrub lands have been variously attributed to facilitation [21, 23, 24], habitat heterogeneity [25], disturbance [26] and localized seed dispersal [27]. In the present study, H. ammodendron (S), H. ammodendron (J), and H. ammodendron (M) all showed aggregated distribution on small scales. First we suggested that micro topography, such as depression, runnel, and sandy mound lead to the high habitat heterogeneity of community, which can leave no doubt that these seed traps played a role in the maintenance of the aggregated structure. Secondly, the interdependence pattern of H. ammodendron (S) and H. ammodendron (J) may improve function to endure drought and sand blown by wind, and increase survival rate. Thirdly, we suggested that water assembly is considered to be the most likely factor that leads to population aggregation. The variation in depression corresponded closely with the distribution of $H$. ammodendron (S) number. In semi-arid and arid areas, water is often the most limiting resource, and small-scale variations in vegetation, soil surface cover, and micro-topography alter the amount of water available to plants $[10,28]$. In addition, soil texture may also play an important role as spacing mechanisms in aggregated pattern form [17].

Wang et al. suggested that species aggregation intensity generally decreased with increasing size classes [29]. Like some previous studies $[7,30]$, we found that relative smaller size classes plants of $H$. ammodendron was more aggregated at small scale than bigger ones in the plot, perhaps because $H$. ammodendron $(\mathrm{S})$ recruitment rates were high and dispersal was rich under condition of environmental heterogeneity. However, the observation that aggregation intensity was weaker in classes with larger size classes even random distribution of $H$. ammodendron $(\mathrm{O})$ may be due to stochastic mortality or strong intra- and/or interspecific competition for resources (water, nutrients, etc.). Our result is consistent with the findings from another study about $H$. ammodendron [11], which report that the species aggregation decreased with increasing size class. This trend suggests that the larger plants competitively inhibit specifics over a larger area of influence than the smaller plants [7]. In this study, the non-random mortality of the H. ammodendron stands driven by intraspecific competition was also observed in other forests $[31,32]$, suggesting that density- 
dependent mortality is a prevailing mechanism in $H$. ammodendron population under drought conditions. Similarly, this provides prevailing evidence suggesting the importance of intraspecific competition in governing the spatial distribution and population dynamics of the plant populations in desert areas.

\section{Intraspecific Associations}

The strongly positive association of $H$. ammodendron at seedlings and juveniles, and juveniles and mature stages at small scales, may result from a facilitative relationship of larger 'nurse plants' providing shelter to small plants. However, the spatial negative association of $H$. ammodendron (S) to $H$. ammodendron (M) at small scales, and $H$. ammodendron $(\mathrm{J})$ to $H$. ammodendron $(\mathrm{O})$ at middle scales may be the result of strong intra- and/or interspecific competition for resources (light, water, nutrients, etc.). In particular, $H$. ammodendron $(\mathrm{O})$ tended to no associations at almost all scales with $H$. ammodendron (S) and $H$. ammodendron (M), indicating the suppression of early stages by later stages. H. ammodendron (S) facilitated the growth of $H$. ammodendron $(\mathrm{O})$ at some smaller scales, whereas $H$. ammodendron (S) was suppressed by $H$. ammodendron (J) at middle scales. H. ammodendron (J) was suppressed by $H$. ammodendron (M) at larger scales, whereas $H$. ammodendron (M) facilitated the growth of $H$. ammodendron $(\mathrm{O})$ at larger scales. The spatial association between different $H$. ammodendron life stages tended to turn from positive to negative if their size differences are enlarged increasingly. The greater the difference in the age of the plants, the weaker the positive correlation $[11,33]$. Therefore, spatial associations of $H$. ammodendron population depend on spatial scales, individual sizes, and plant interaction relationships.

\section{Implications for Population Management}

Micro-topography creates biotic heterogeneities (e.g., seed tree abundance, intraspecific competition) and abiotic heterogeneities (e.g., soil organic, salt, water), which act as a filter for seed availability, seedling germination, and establishment in arid and semi-arid environments [27, 34]. Thus, for H. ammodendron with limited seed dispersal distance, seed availability may be the main limiting factor for recruitment in severely heterogeneities topography areas and result in competitive relation and aggregated patterns in desert areas [11]. Furthermore, field observations also confirmed that almost all recruits were observed in low severity burned areas. In addition, drought is an important disturbance in the desert community, which can change vegetation dynamics $[10,34]$. Because community succession is a long-term process where initial conditions can be critically important, plant recruitment after drought severity provides a critical observational window to predict future spatial patterns and stand dynamics $[11,27]$. In the present, due to the sandy soils of our study area, severely continued drought may be likely to kill the seedling meristems or even seedlings of $H$. ammodendron, hence ultimately limiting post-drought seedling recruitment poten- tial. Thus, artificial regeneration and afforestation using micro-topography may be to promote ecological restoration of the degraded $H$. ammodendron ecosystem in lowseverity drought years.

Sustainable forest management in arid and semi-arid areas has long been interested in managing interspecific relations dominated by desert woody species, but intraspecific competition also is a very important question [34]. In this paper, we showed that intraspecific competition was an important process in four different life stages (seedlings, juveniles, mature, overmature) in a $H$. ammodendron population in the Ecotone desert-oasis of South Junggar Basin. The results of our spatial analyses suggest that minimizing the intraspecific competition between conspecific plants will likely increase the survivorship of desert plants. The maintenance of the plant community will not only increase the diversity in stand composition but also will reduce the intensity of intraspecific competition and consequent mortality [35]. Furthermore, the stand density of $H$. ammodendron must be optimized to maintain a plant growth that compensates the loss due to competition under drought conditions.

\section{Conclusions}

The present study provides further evidence of $H$. ammodendron population dynamics and intraspecific competition at different life stages in the desert-oasis ecotone of south Junggar Basin, NW China. The species functional traits (regeneration, seed dispersal ability, etc.), water assemble, soil texture, and environmental heterogeneity have been considered to be a primary factor controlling the aggregated distribution of the $H$. ammodendron population. The spatial associations of $H$. ammodendron population in Junggar Basin not only varied with life stages, but also with spatial scales.

\section{Acknowledgements}

Our research is supported by the National Basic Research Program of China [2010CB951003] and the Cultivation Program of Young Scientific and Technological Talent of Shihezi University [2013ZRKXYQ11]. The authors thank all those who provided helpful suggestions and critical comments on this manuscript and anonymous reviewers. The authors also wish to thank Dr. Yaning Chen for his assistance in the fieldwork.

\section{References}

1. HUSTON M.A., DEANGELIS D.L. Size bimodality in monospecific populations: A critical review of potential mechanisms. Am. Nat. 129, (5), 678, 1987.

2. WEINER J., DAMGAARD C. Size-asymmetric competition and size-asymmetric growth in a spatially explicit zone of influence model of plant competition. Ecol. Res. 21, (5), 707, 2006. 
3. METSARANTA J.M., LIEFFERS V.J. Inequality of size and size increment in Pinus banksiana in relation to stand dynamics and annual growth rate. Ann. Bot. 101, (4), 561, 2008.

4. ERFANIFARD Y., FEGHHI J., ZOBEIRI M., NAMIRANIAN N. Comparison of two distance methods for forest spatial pattern analysis. J. Appl. Sci. 8, (1), 152, 2008.

5. POMMERENING A. Approaches to quantifying forest structures. Forestry. 75, (5), 305, 2002.

6. SKOV F., SVENNING J.C. Predicting plant species richness in a managed forest. Forest. Ecol. Manag. 180, (3), 583, 2003.

7. CHENG X.Q., HAN H.R., KANG F.F., SONG Y.L., LIU K. Point pattern analysis of different life stages of Quercus liaotungensis in Lingkong Mountain, Shanxi Province, China. Journal of Plant Interactions. 9, (1), 233, 2014.

8. LI L., WEI S.G., HUANG Z.L., YE W.H., CAO H.L. Spatial patterns and interspecific associations of three canopy species at different life stages in a subtropical forest, China. J. Integr. Plant Biol. 50, (9), 1140, 2008.

9. WU Z.Y. Vegetation of China. Science Press, China, 1980 [In Chinese].

10. ZOU T., LI Y., XU H., XU G.Q. Responses to precipitation treatment for Haloxylon ammodendron growing on contrasting textured soils. Ecol. Res. 25, (1), 185, 2010.

11. SONG Y.Y., LI Y.Y., ZHANG W.H. Analysis of spatial pattern and spatial association of Haloxylon ammodendron population in different developmental stages. Acta Ecologica Sinica. 30, (16), 4317, 2010 [In Chinese].

12. NANAMI S., KAWAGUCHI H., YAMAKURA T. Spatial pattern formation and relative importance of intra and interspecific competition in codominant tree species, Podocarpus nagi and Neolitsea aciculate. Ecol. Res. 26, (1), 37, 2011.

13. WIEGAND T., MOLONEY K.A. Rings, circles and nullmodels for point pattern analysis in ecology. Oikos. 104, (2), 209, 2004.

14. STOYAN D., PENTTINEN A. Recent applications of point process methods in forestry statistics. Stat. Sci. 15, (1), 61, 2000.

15. GETZIN S., WIEGAND T., WIEGAND K., HE F.L. Heterogeneity influences spatial patterns and demographics in forest stands. J. Ecol. 96, (4), 807, 2008.

16. TAKAHASHI K., HONIMA K., VETROVA V.P., FLORENZEV S., HARA T. Stand structure and regeneration in a Kamchatka mixed boreal forest. J. Veg. Sci. 12, (5), 627, 2001.

17. ZHANG Z.H., HU G., ZHU J.D., LUO D.H., NI J. Spatial patterns and interspecific associations of dominant tree species in two old-growth karst forests, SW China. Ecol. Res. 25, (6), 1151, 2010.

18. HOU J.H., MI X.C., LIU C.R., MA K.P. Spatial patterns and associations in a Quercus-Betula forest in northern China. J. Veg. Sci. 15, (3), 407, 2004.

19. SI L.M., LIU T., LIU B., LI L. A comparative study on reasons of degenerated of Haloxylon ammodendron population in the western part of Gurbantunggut desert. Acta Ecologica Sinica. 31, (21), 6460, 2011 [In Chinese].
20. LI L., HUANG Z.L., YE W.H., CAO H.L., WEI S.G. , WANG Z.G., LIAN J.Y., SUN I.F., MA K.P., HE F.L. Spatial distributions of tree species in a subtropical forest of China. Oikos. 118, (4), 495, 2009.

21. TIRADO R., PUGNAIRE F.I. Shrub spatial aggregation and consequences for reproductive success. Oecologia. 136, (2), 296, 2003

22. RAYBURN A.P., SCHIFFERS K., SCHUPP E.W. Use of precise spatial data for describing spatial patterns and plant interactions in a diverse Great Basin shrub community. Plant Ecol. 212, (4), 585, 2011.

23. PUGNAIRE F.I., ARMAS C., MAESTRE F.T. Positive plant interactions in the Iberian southeast: mechanisms, environmental gradients, and ecosystem function. J. Arid Environ. 75, (12), 1310, 2011.

24. RAYBURN A.P., WIEGAND T. Individual species-area relationships and spatial patterns of species diversity in a Great Basin, semi-arid shrubland. Ecography. 35, (4), 341, 2012.

25. PERRY G.L.W., ENRIGHT N.J., MILLER B.P., LAMONT B.B. Nearest-neighbor interactions in species-rich shrublands: the roles of abundance, spatial patterns and resources. Oikos. 118, (2), 161, 2009.

26. SEIFAN M., KADMON R. Indirect effects of cattle grazing on shrub spatial pattern in a Mediterranean scrub community. Basic Appl. Ecol. 7, (6), 496, 2006.

27. SCHURR F.M., BOSSDORF O., MILTON S.J., SCHUMACHER J. Spatial pattern formation in semi-arid shrubland: a priori predicted versus observed pattern characteristics. Plant Ecol. 173, (2), 271, 2004.

28. SHENG J.H., QIAO Y.X., LIU H.Y. A study on the root system of Haloxylon Aammodendron (C. A. Mey.) Bunge. Acta Agrestia Sinica. 12, (2), 91, 2004 [In Chinese].

29. WANG X.G., YE J., LI B.H., ZHANG J., LIN F., HAO Z.Q. Spatial distributions of species in an old-growth temperate forest, northeastern China. Can. J. For. Res. 40, (6), 1011, 2010.

30. SEIWA K., MIWA Y., SAHASHI N., KANNO H., TOMITA M., UENO N., YAMAZAKI M. Pathogen attack and spatial patterns of juvenile mortality and growth in a temperate tree, Prunus grayana. Can. J. For. Res. 38, (9), 2445, 2008.

31. DUNCAN R.P. Competition and the coexistance of species in amixed podocarp stand. J. Ecol. 79, (4), 1073, 1991.

32. HE F., LEGENDRE P., LAFRANKIE J.V. Distribution patterns of tree species in a Malaysian tropical rain forest. J. Veg. Sci. 8, (1), 105, 1997.

33. YANG H.X., ZHANG J.T., WU B., LI X.S., ZHANG Y.Y. Point pattern analysis of Artemisia ordosica population in the Mu Us Sandy Land. Journal of Plant Ecology. 30, (4), 563, 2006 [In Chinese].

34. CHU G.M., WANG M., ZHANG S.X. Spatial patterns and associations of dominant woody species in desert-oasis ecotone of South Junggar Basin, NW China. Journal of Plant Interactions. 9, (1), 738, 2014.

35. GRAY L., HE F.L. Spatial point-pattern analysis for detecting density-dependent competition in a boreal chronosequence of Alberta. Forest. Ecol. Manag. 259, (1), 98, 2009. 
